\title{
Subject Index Vol. 73, 2006
}

Digestion

Acute hepatitis 167

- pancreatitis 228

Akt kinase/protein kinase B 75

5-Aminosalicylates 11

Antacids 198

Antibiotic resistance 101

Antroduodenal manometry 151

Apoptosis 75

Aspirin 54, 124

Audit process 189

Biliary cancer 228

Biliopancreatic reflux 228

Bleeding 198

Budesonide 60

cagA gene 47

Calcium, intracellular 249

Carcinoma in situ 160

Caspase 75

Chemoprevention 54

Chemospecific visceral sensitivity 151

Cholecystokinin octapeptide 249

Cholecystolithiasis 69

Chronic hepatitis C 210

Clarithromycin 101

Coeliac disease 20

Colonic inflammation 151

Colonoscopy screening 84

Colorectal cancer 84

- -, prevention 11

Complementary medicine 167

Corticosteroids 107

COX-2 expression 124

C-reactive protein, high sensitivity 205

Crohn's disease 1, 40, 107, 205, 210, 237

- $\quad$ and ulcerative colitis 25

- -, appropriateness of care 237

- -, practice guidelines 237

- $\quad$, , quality of care 237

- -, therapy 237

Cyclooxygenase-2 inhibitor SC236 75

Cytochrome $c 75$

Dietary supplement 167

DNA microarray analysis, high-density 89

Dual-energy X-ray absorptiometry, bone mineral density measurement 40

Duodenal ulcer 47
Supplement issue No. 1 has its own Subject Index

Endoscopy 160

Enteropathy-associated T-cell lymphoma 20

EPACT panel 237

Erosive reflux esophagitis 171

Esomeprazole 178

Esophageal acid exposure 171

- cancer 160

Food hypersensitivity 111

Functional heartburn 218

Gallbladder function 69

Gastric cancer 47, 54

- epithelial cell, rat 89

- mucosa 69

- strips 249

- ulcer 32

Gastroesophageal reflux disease 171, 218

Gastrointestinal pathology 1

Gastroscopy 189

Glucagon-like peptide-1 142

Granulation tissue 32

Head and neck cancer 160

Helicobacter pylori 47, 69, 101, 116, 178

- - eradication 101

Hepatitis C virus 210

Herbal toxicity 167

Hyperlipidemic pancreatitis 259

Hypersensitive esophagus 218

Hypersensitivity, rectal 133

Hypertriglyceridemia 259

Hypocompliance, rectal 133

Hypoxia 89

Immune response 116

Indomethacin 32

Ineffective esophageal motility 171

Inflammatory bowel disease 11,25 , 151

- - -, chronic 1

Informed consent 189

Inositol 1,4,5-triphosphate receptor 249

Intestinal allergen provocation test 111

Irritable bowel syndrome 133

Lactobacillus acidophilus 107

Liver abscess 107
Magnetic resonance imaging 111

Mast cells 116

Meta-analysis 178

Misoprostol 32

Motility 124

Mucosal injury 89

Natriuresis 142

NOD2/CARD15 mutation 107

Omeprazole 32

Osteopenia 40

Osteoporosis 40

Pancreaticobiliary maljunction 228

Pancreatobiliary reflux 228

Paraffin-embedded section 47

Patch clamp technique 249

Pegylated interferon 210

Peptic ulcer 178, 198

Plasmapheresis 259

Prostaglandin 124

Proton pump inhibitors 198, 218

RAND Appropriateness Method 237

Randomized controlled trials 178

Refractory sprue 60

Reoxygenation 89

Resource implication 84

Ribavirin 210

Risk factors, low bone mineral density 40

SC236 cyclooxygenase-2 inhibitor 75

Sucralfate 198

Symptoms, irritable bowel syndrome 133

T cell antigen 60

- _ receptor gene rearrangement 60

6-Thioguanine 25

Thiopurine 25

Thirst regulation 142

Ulcerative colitis 1, 40, 205

- jejunoileitis 20

Ultrasound 111 